\title{
Una herramienta de ayuda para la decisión fue útil en pacientes con diabetes
}

\author{
A decision-making-tool was useful in a diabetic population
}

\section{Objetivo}

Evaluar en pacientes con diabetes tipo 2 el efecto de una herramienta de ayuda para la toma de decisiones (AD) para el comienzo del tratamiento con estatinas en relación a: su conocimiento del problema, sus conflictos, la acción resultante y su adherencia al fármaco.

\section{Diseño}

Ensayo clínico aleatorizado en "cluster"*, con asignación ciega de los participantes del estudio y seguimiento a tres meses.

Lugar

Mayo Clinic, Minnesota, EE.UU.

\section{Pacientes}

98 pacientes con diabetes tipo 2 .

\section{Intervención}

Se comparó la $A D$ con la hoja informativa estándar de manejo de colesterol de la Clínica Mayo y analizando los resultados por intención de tratar. La AD se personalizó para cada paciente e
Weymiller A y col. Arch Intern Med. 2007; 167:1076-1082

incluyó la presentación del riesgo cardiovascular a los diez años, la reducción del riesgo absoluto con el uso de estatinas y las desventajas de las mismas.

\section{Resultados principales}

Los pacientes prefirieron el uso de la herramienta de ayuda para la decisión (OR 2,8; IC95\% 1,2 a 6,9). Quienes la habían recibido $(n=52)$ estaban mejor informados $(2,4$ puntos de diferencia de un total de 9; IC95\% 1,2 a 6,9) conocían mejor su riesgo cardiovascular (OR 22,4; IC95\% 5,9 a 85,6) y la reducción potencial del riesgo absoluto con estatinas (OR 6,7; IC95\% 1,5 a 3,3); y tuvieron menos conflicto en la decisión (diferencia -10,6 puntos; IC95\% -15,4 a -5,9 sobre una escala de 100). De los 33 pacientes en el grupo intervención que estaban tomando estatinas, dos reportaron que se habían olvidado una dosis o más en la última semana, contra seis de los 29 del grupo control (OR 3,4; IC95\% 1,5 a 7,5).

\section{Conclusiones}

La decisión ayudada podría haber afectado favorablemente la adherencia a estas drogas.

\section{Comentario}

La enfermedad cardiovascular (CV) es la principal causa de muerte en los países desarrollados y en muchos de los en vías de desarrollo. Varios ensayos clínicos ${ }^{1,2,3,4}$ demostraron que el tratamiento regular y durante largos períodos con estatinas reduce la morbimortalidad en prevención primaria y secundaria. Los resultados del "Heart Protection Study" hicieron que se recomiende una meta de LDLc menor a $70 \mathrm{mg} / \mathrm{dL}$ en pacientes de muy alto riesgo; ya que en dicho estudio se observó una relación lineal entre la reducción seríca de LDLc y la del riesgo relativo de eventos coronarios, efecto que sería independiente del nivel basal de LDLc, ya que incluso siendo este menor a $100 \mathrm{mg} / \mathrm{dL}$ se reportó una disminución de $30 \%$ en el riesgo en todos los eventos.

Si bien sería eficaz tratar con estatinas a toda la población hipercolesterolémica, sería una estrategia poco costo-efectiva. Por tal motivo es prudente estratificar correctamente a los pacientes, ya que la evidencia sugiere que se beneficiarían más los pacientes con riego cardiovascular alto.

Como lo muestra un estudio canadiense de $2002^{5}$, la adherencia al tratamiento con estatinas es baja: $40,1 \%$ de los pacientes de alto riesgo cardiovascular y $25,4 \%$ de los de bajo riesgo. Vale aclarar que el término anglosajón adherencia implica que el paciente, desde un rol activo y habiendo comprendido las ventajas y desventajas de sus potenciales conductas, acepta y pone en práctica las recomendaciones del equipo de salud. No es sinónimo de cumplimiento, ya que este último concepto sólo se refiere a una conducta pasiva en el sentido de aceptar las indicaciones médicas.

\section{Conclusiones de la comentadora}

La discusión con el médico y la comprensión del riesgo cardiovascular personal a diez años, condujeron a que los pacientes adhirieran con menos conflicto y más rapidamente a las recomendaciones terapéuticas. Si bien fue un estudio bien diseñado presenta las limitaciones de haber enrolado pacientes que ya recibían estatinas, que habían tenido educación terapéutica previa, que eran referidos a un nivel especializado de atención y de haberlos seguido sólo durante tres meses.

Sin embargo, la creciente evidencia a su favor estimula al equipo de salud el uso de herramientas sencillas para ayudar a los pacientes a tomar las mejores decisiones compatibles con sus valores. De todos modos, serán necesarias nuevas investigaciones que enrolen pacientes con menor educación terapeútica previa y con seguimiento más prolongado para esclarecer la efectividad de este tipo de herramienta a nivel local.

Adriana Primerano [ Servicio de Medicina Familiar y Comunitaria. Hospital Italiano de Buenos Aires. adriana.primerano@hospitalitaliano.org.ar ]

Primerano A. Una herramienta de ayuda para la decisión fue útil en pacientes con diabetes. Evid. actual. práct. ambul; $11(2)$ : 42 , Mar-Abr.2008. Comentado de Weymiller A, y col. Statin Choice Randomized Trial. Arch Intern Med. 2007; 167:1076-1082. PMID: 17533211

\section{Referencias}

1. Heart Protection Study Collaborative Group. MRC/BHF Heart Protection Study of cholesterol lowering with simvastatin in 20536 high-risk individuals: a randomised placebo controlled trial. Lancet. 2002; 360: 7-22. HPS.

2. Shepherd J, et al on behalf of the PROSPER study group. Pravastatin in elderly individuals at risk of vascular disease (PROSPER): a randomised controlled trial Lancet. 2002; 360: 1623-30. PROSPER.

3. Sever $\mathrm{P}$ et al. for the ASCOT Investigators. Prevention of coronary and stroke events with atorvastatin in hypertensive patients who have average or lower-than-average cholesterol concentrations, in the Anglo-Scandinavian Cardiac Outcomes Trial一Lipid Lowering Arm (ASCOT- LLA): a multicentre randomised controlled trial. Lancet 2003; 361: 1149-58 ASCOT-LLA.

4. Cannon C, et al. Comparasion of Intensive and Moderate Lipid Lowering with Statins after Acute Coronary Syndromes. N Engl J Med 2004;350:1495-504 PROVE-IT.

5. Jackevicius C. Adherence With Statin Therapy in Elderly Patients With and Without Acute Coronary Syndromes. JAMA 2002; 288:462-467. 